\title{
Hybrid Particle Swarm Optimization and k-Means Clustering for Education Quality Mapping
}

\author{
Muhammad Lintang Cahyo \\ Department of Information System \\ Diponegoro University \\ Semarang, Indonesia
}

\author{
Jatmiko Endro Suseno \\ Department of Physics \\ Diponegoro University \\ Semarang, Indonesia
}

\author{
Oky Dwi Nurhayati \\ Department of Informatic \\ Diponegoro University \\ Semarang, Indonesia
}

\begin{abstract}
Quality mapping on education can be useful information to evaluate how well the quality of education is attained in each school. Solutions to overcome this problem required an information system for mapping the quality of education based on the five categories of quality achievement levels set by the government of Indonesia. This study was conducted using 200 school datasets in the city of Semarang. the study used the test parameters of input 20 particles, 40 particles, 80 particles and 160 particles. parameters were tested using PSO and PSO + k-Means methods. As a result, the use of the 20 particles parameter provides an optimal solution in grouping data at a central point
\end{abstract}

\section{General Terms}

Education quality mapping

\section{Keywords}

Information system, clustering, particle swarm optimization, k-Means clustering

\section{INTRODUCTION}

Mapping of the quality of education is a process that seeks to ensure the implementation of the quality of education. The function of quality assurance of education is aimed at the quality of schools in accordance with the criteria that have been determined by the government. Implementation of the basic standard of education in quality mapping in schools can be useful information for evaluating the quality of education achievement in each school. The solution to solve this problem requires an information system to categorize the quality of education based on the five categories of quality achievement levels established by the government in Indonesia as one of the benchmarks in evaluating school quality achievement. One scientific method capable of grouping large data into each category can use hybrid kMeans and particle swarm optimization (PSO) methods.

Clustering is one statistical analysis that aims to group data into each cluster, used in assisting data modeling [1]. Clustering in the information system application plays a role in facilitating grouping in information retrieval [2]. Clustering can be used as a tool for preprocessing on other algorithms [3].

k-Means is able to build an empirical data classification [4] and can also be applied in grouping heterogeneous data into more homogeneous sub data [5]. The k-Means algorithm can be applied to large amounts of data. The approach uses an entire set of data divided into smaller sets [6].

The k-Means algorithm is one of the best and very efficient Clustering algorithms but its performance is very sensitive to cluster initialization with optimal results. k-Means will be able to find the right central point if the initial generation of the central point made with the random value is close to the final solution of the cluster center. If the beginning of the central point is far from the final solution of the cluster center then it is likely that the clustering result will be incorrect [7]. The solution to solve this problem is with a PSO hybrid. PSO algorithm produces optimization based on population data. The algorithm is able to find the optimal solution, or nearoptimal on the problem of quantitative and qualitative data. PSO and k-Means hybrid algorithms produce better results than without hybrid [8].

The PSO + k-Means method aims to classify school education standard data into five categories: first level education standard, second level education standard, third level education standard, fourth level education standard and fifth level education standard. The application of hybrid k-Means and PSO in this research is expected to develop an effective quality education information system for management, decision making in guaranteeing and improvement of education quality.

\section{RELATED WORK}

Implementation of k-Means Clustering Algorithm and PSO is able to partition a number of clusters that have been determined. The PSO algorithm is used as a search for the best initial centroid values for each cluster to be established [9]. The k-Means and PSO method approach can shorten computing time. The PSO-coupled algorithm with k-Means Algorithm shows a high degree of classification accuracy with a slight degree of classification error compared with regular kMeans [10].

The grouping of PSO and k-Means in data clustering is capable of performing rapid data groupings [11]. Basically PSO is used to improve clusters formed by k-Means [12]. As the performance of PSO is able to initialize the initial value on global search. When it is close to the optimal value, then switch to the k-Means algorithm as a clustering step. This suggests that hybrid algorithms are better at clustering data [13].

\section{RESEARCH METHODOLOGY}

The research methodology used to solve the problem of educational mapping is described as follows :

\section{1 k-Means Algorithm}

The k-Means algorithm is a best method of partitioning in data groupings. The data that have the same characteristics are grouped in one cluster, and data that have different characteristics are grouped in another cluster. Group of data based on the closest distance to each other according to 
Euclidean distance. The value of $\mathrm{k}$ is required as an input parameter and a partition in a set of objects $n$ of cluster $k$. The average value of the set of objects is taken as the resemblance of parameters to form clusters [14].

The k-Means algorithm classifies the object $\mathrm{n}$ into cluster $k$ by observing each of the closest middle values into the cluster. The resembling data resides in the same cluster and the unequal data resides in another cluster. Each cluster is associated with the centroid that is the center of the cluster [15]. The steps of clustering with the k-Means method are as follows [1]:

a. Determine the number of $k$ values to be established. The number of $k$ values is five clusters based on the five categories of standar education.

b. Determination of the value of the center of the early cluster (centroid). Randomly determining the value of the centroid that will be the center of the cluster as much as the number of $k$ according to the first stage.

c. Determine the distance between the weights on each data that is not the cluster center (centroid) with the weight of each data in each cluster center using the euclidean distances formula [16].

$$
d_{\left(x_{j}, c_{i}\right)}=\sqrt{\sum \frac{N}{j=1}\left(x_{j, a}-c_{i, a}\right)^{2}}
$$

caption :

$$
\begin{aligned}
& d_{\left(x_{j}, c_{i}\right)}=\text { distance of data to the cluster center } \\
& N=\text { the amount of data } \\
& x_{j, a}=\text { data on attribute to } j \text { on record to } a \\
& c_{i, a}=\text { data at centroid value to } i \text { record to } a
\end{aligned}
$$

d. The shortest distance determination.

After getting the distance between data with the centroid, then specify $d$ that has minimum value on each data to become cluster member.

centroid value $=\sum \frac{c_{i}}{c_{k}}$

caption :

$c_{i}=$ record to $i$ against each data selected to be a cluster member at the stage 4 .

$c_{k}=$ the number of cluster members formed in stage 4 .

e. Termination of iteration.

f. Repeat steps 3-6 until the centroid value of each cluster member is unchanged. So get clusters that contain similar data.

\subsection{Particle Swarm Optimization}

PSO is a stereotypical optimization method that represents the solution of problems in the form of particles[17]. The PSO algorithm is inspired by the social behavior of animals such as a collection of birds in a population (swarm). Social behavior consists of individual actions and the influence of other individuals in a group. The particles show a bird in a flock of birds. Each individual or particle behaves by using its own intelligence and also influenced by the behavior of its collective group.

The PSO algorithm is guided by personal experience or Particle Best (Pbest), the overall experience of the swarm particles set of Global Best (Gbest) and the movement of velocity particles to determine the next position in the search space. Acceleration of particles is influenced by two factors: $c 1$ and $c 2$, and two random values ranging from 0 to 1 . The process of particle position displacement is multiplied by the inertia factor $w$ varying in the range of $w_{-} \min$ to $w_{-} \max$. The initial velocity of the population $v=\left[v_{1}, v_{1}, \ldots, n\right]^{T}$, where $T$ is the transpose operator. So the velocity of each particle $\quad X_{i}(i=1,2, \ldots, N) \quad$ becomes $v_{i}=\left[v_{i, 1}, v_{i, 1}, \ldots, v_{i, n}\right][18]$. The steps in using PSO algorithm are as follows:

a. Spesify parameter, $w, c_{1}, c_{2}, i$, maxite

Caption :

$w=$ inertia factor value

$c_{1}=$ learning rate for particle

$c_{2}=$ learning rate for swarm

$i=$ number of particle

maxite $=$ maximum iteration

b. Initialize the random (random) position of each particle $x i$, and the velocity of each particle $v i$.

Caption :

$x_{i}=$ Position of particles (position)

$y_{i}=$ Particle velocity (velocity)

$i=$ index of particles in one population, $i \in\{1,2, \ldots, n\}$

c. Determine the initial iteration $k=1$.

d. Calculates the $F_{i}$ particle fitness value on the initial iteration based on the formula below and find the best index for particle $b$.

$F_{i}^{k}=f\left(x_{i}^{k}\right)$.

and do a fitness value calculation on the next iteration of the entire population (swarm) to get the particle $b 1$.

$$
F_{i}^{k+1}=f\left(x_{i}^{k+1}\right)
$$

Caption:

$b=$ the best particle index in a population

$F_{i}^{k}=$ the value of the objective function of the particles in one population at the iteration to $k$

$f\left(x_{i}^{k}\right)=$ evaluation of objective function

e. Select

Pbest $_{i}^{k}=x_{i}^{k}$ and Gbest $^{k}=x_{b}^{k}$

Caption :

Pbest $_{i}^{k}=$ personal best (local) ever achieved

particle to in one population until iteration to $k$

Gbest $^{k}=$ global best ever achieved by one population until iteration to $k$

f. Calculate the value of inertia vector using the equation

$w=w_{\max }-k \times\left(w_{\max }-w_{\min }\right) /$ Maxite

g. Update velocity and position values for particle in whole population (swarm) :

$$
\begin{aligned}
& v_{i, j}^{k+1}=w \times v_{i, j}^{k}+c_{1} \times r_{1} \times\left(\text { Pbest }_{i, j}^{k} \times x_{i, j}^{k}\right) \\
& +c_{2} \times r_{2 \times}\left(\text { Gbest }_{j}^{k}-x_{i, j}^{k}\right) \\
& x_{i, j}^{k+1}=x_{i, j}^{k}+v_{i, j}^{k+1}
\end{aligned}
$$$$
\text { and }
$$

h. Update Pbest populations 
if $F_{i}^{k+1}$ then Pbest $_{i}^{k+1}=x_{i}^{k+1}$

else Pbest $_{i}^{k}$

Pbest $_{i}^{k+1}=$ (9)

i. Update Gbest population

if $F_{b 1}^{k+1}<F_{b}^{k}$ then Gbest $^{k+1}=$ Pbest $_{b 1}^{k+1}$

and

set $b=b 1$ else Gbest ${ }^{k+1}=$ Gbest $^{k}$

i. If $k<$ maxite then $k=k+1$ and go back to step 6. If $k>$ maxite then go to step 11 .

j. The calculation cycle of velocity and position will continue to be repeated until iteration is complete. When iteration is over, the best particles come out as the optimum solution Gbest ${ }^{k}$.

\subsection{Hybrid PSO + k-Means}

Hybrid-PSO algorithm is a hybrid of k-Means and PSO methods of clustering. In this, k-Means is executed once and the results of k-Means are used to seed one of the particles in PSO clustering algorithm. Then PSO algorithm is executed.

1) Algorithm for hybrid PSO clustering

1. Select number of particle

2. Execute k-Means on the data and assign the calculated centroid to one particle

3. Initialize other particle to have randomly selected $N_{c}$ cluster centroid.

4. For $i$ in range maxite :

a. For $j$ in range number of particles :

i. For each data vector :

a) Calculated the Euclidean distance to all cluster centroid

b) Assign the data vector to the cluster such that the Euclidean distance is minimum

ii. Calculate the fitness function using equation 3 .

b. Update local best position using equation 5 .

c. Update the global best position as the position particle which minimize the fitness function.

d. Update the cluster centroid with equation 7 and 8.

\section{DESIGN OF RESEARCH}

\subsection{Material and Tools}

The tools and materials used in this research are 200 school datasets with 8 criteria. the data is processed using PSO and K-Means methods. we use secondary data which located in semarang city, Indonesia. Data processing is done by using matlab software as the first step in doing parameter test.

\subsection{Research Procedure}

The research procedure of PSO + k-Means implementation is shown in Figure 1.

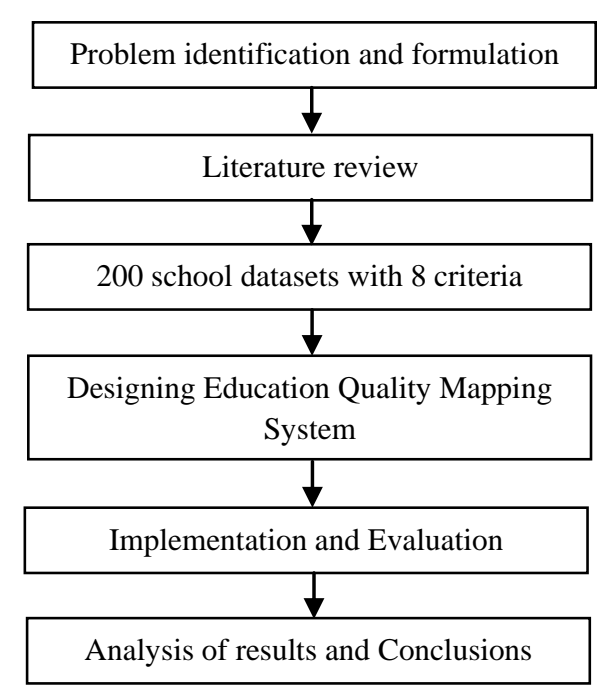

Fig 1: Research Procedure

\subsection{Physical Data Model}

\subsubsection{User Login Table}

User login table used to store users who can login, as shown

in Table 1.

Table 1. Login User

\begin{tabular}{|l|l|l|l|}
\hline Attribute & Type & Length & Detail \\
\hline Id & Integer & 10 & Primary key \\
\hline Username & Varchar & 20 & Name of user \\
\hline Password & Varchar & 20 & passcode \\
\hline
\end{tabular}

4.3.2 Data Table

Data tables are used to store school identity and scores obtained from secondary data.

Table 2. Data Table

\begin{tabular}{|l|l|l|l|}
\hline Attribute & Type & Length & Detail \\
\hline Id & Integer & 15 & Primary key \\
\hline School name & Varchar & 20 & School identity \\
\hline First value & Float & 5 & Standar score \\
\hline Second value & Float & 5 & Standar score \\
\hline Third value & Float & 5 & Standar score \\
\hline Fourth value & Float & 5 & Standar score \\
\hline Fifth value & Float & 5 & Standar score \\
\hline Sixth value & Float & 5 & Standar score \\
\hline Seventh value & Float & 5 & Standar score \\
\hline Eight value & Float & 5 & Standar score \\
\hline
\end{tabular}

\subsubsection{Setting Table}

Setting table is used as the input parameter setting. 
Table 3. Setting Table

\begin{tabular}{|l|l|l|l|}
\hline Attribute & Type & Length & Detail \\
\hline Id & Integer & 5 & Primary key \\
\hline C1 & Float & 5 & Cognitive 1 \\
\hline C2 & Float & 5 & Cognitive 2 \\
\hline Swarm & Float & 5 & Swarm size \\
\hline Number of k & Float & 5 & Number of cluster \\
\hline w & Float & 5 & Weight score \\
\hline
\end{tabular}

\subsubsection{Clustering Result table}

this table is used as showing the output of clustering results.

Table 4. Result Table

\begin{tabular}{|l|l|l|l|}
\hline Attribute & Type & Length & Detail \\
\hline Id & Integer & 5 & Primary key \\
\hline School name & Float & 5 & School name \\
\hline Level score & Float & 5 & score \\
\hline
\end{tabular}

\section{EXPERIMENTAL RESULT}

\subsection{Information System Framework}

200 The data on school education value is processed using PSO method to find the best centroid value. The best centroid results are processed using K-Means method for value grouping process. the results are grouped into five level categories.

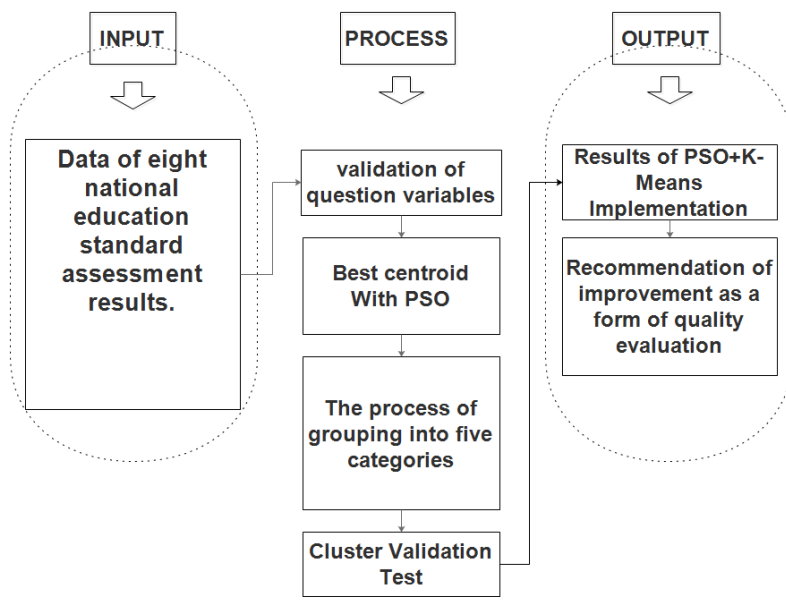

Fig 2: Information System Framework

In this case the K-Means method requires accurate initial centroid values in order to properly cluster the data into each cluster. The PSO algorithm can solve the problems in the KMeans Algorithm. Hybrid PSO + k-Means testing was done using 200 school data in which there were 8 assessment criteria. To test the system, tested by experimenting with several particle inputs on the PSO, 20 particles, 40 particles, 80 particles and 160 particles. In addition to the particle parameter input, other parameters used as trials in this case are inertia weigth (w), c1, c2, and initial velocity. The input parameters used in testing the ability of the methods in the dataset, namely: $\mathrm{w}=1.5, \mathrm{c} 1=1.5, \mathrm{c} 2=0.7$. Figure 3 below Explains the optimal value search results by using the inputted parameter in the form of 20 particles. The population of scattered data is almost gathered at one optimal point. Some other data still have not reached to the optimum point.

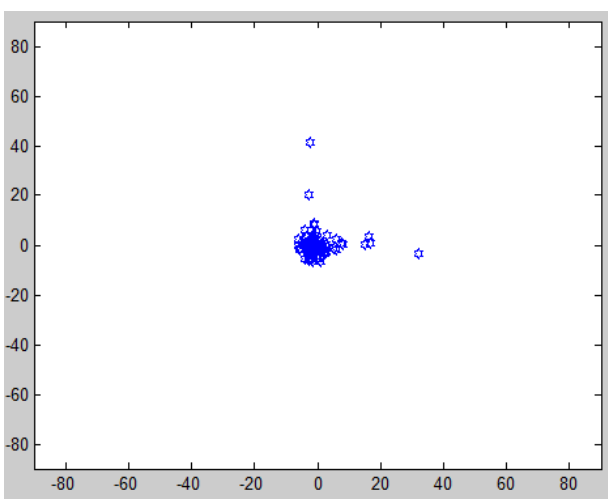

Fig 3: PSO with parameter of 20 particles

In figure 4 and figure 5 below using the 40 particles and 80 particles parameters, the optimal point search for the whole data is almost towards the optimum point, there are some data populations that have not reached the optimum point.

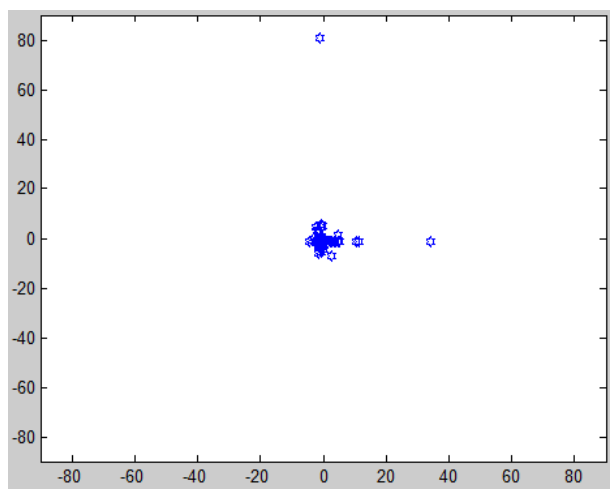

Fig 4: PSO with parameter 40 particles

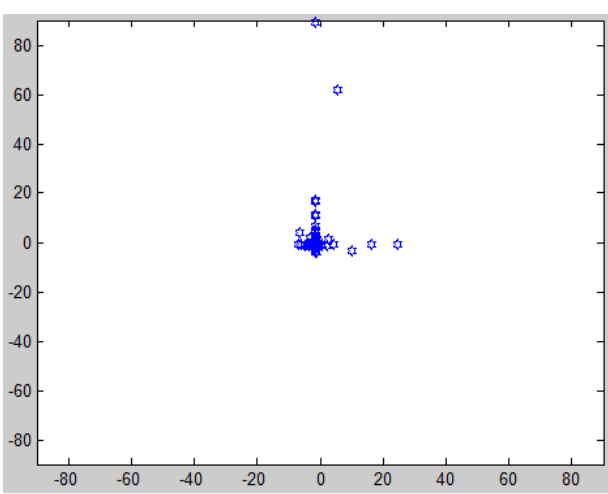

Fig 5: PSO with parameter 80 particles

Figure 6 below produces a data population centered on a single point. There are some populations of data that are not centered on an optimal point, but the results obtained are good compared to some previous experiments. 


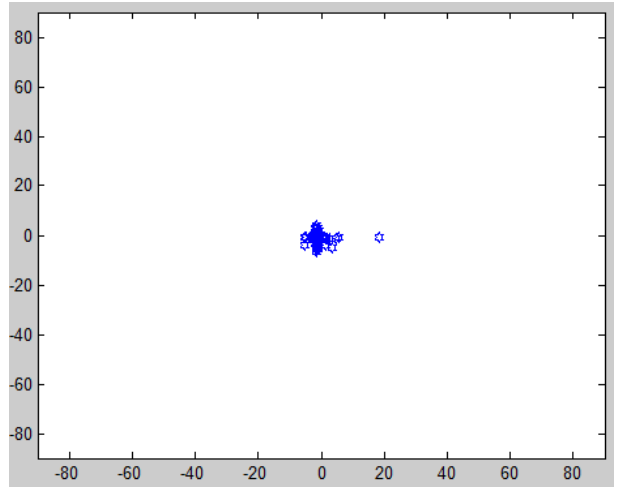

Fig 6: PSO with parameter 160 particles

Figure 7 below describes the implementation of the PSO + KMeans hybrid. Hybrid results by using different particle parameters, resulting in different data groupings. The output of PSO + K-Means method is not so different from the results obtained by using PSO method. The accuracy produced using PSO + K-Means method is different. The results are more accurate using PSO+K-Means method than without hybrid.

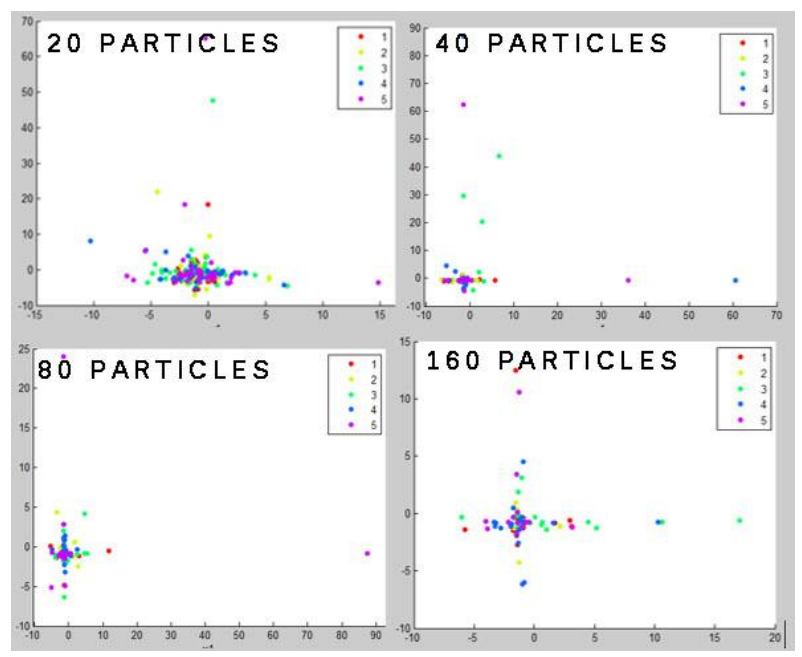

Fig 7: PSO+K-Means with different parameter

Figure 8 below describes the fitness values used to find the optimal value in the data population. Fitness value using the PSO method there is a decrease in the value of the use of 40 particles parameters and increased in parameters 80 and 160 particles. In PSO, the fitness value is very relative. When the use of hybrid PSO + K-Means in the search for the optimal value to clustering data, there is an increase in fitness value when adding particle parameters. The smaller the fitness value, then the search for the optimal value will be better.

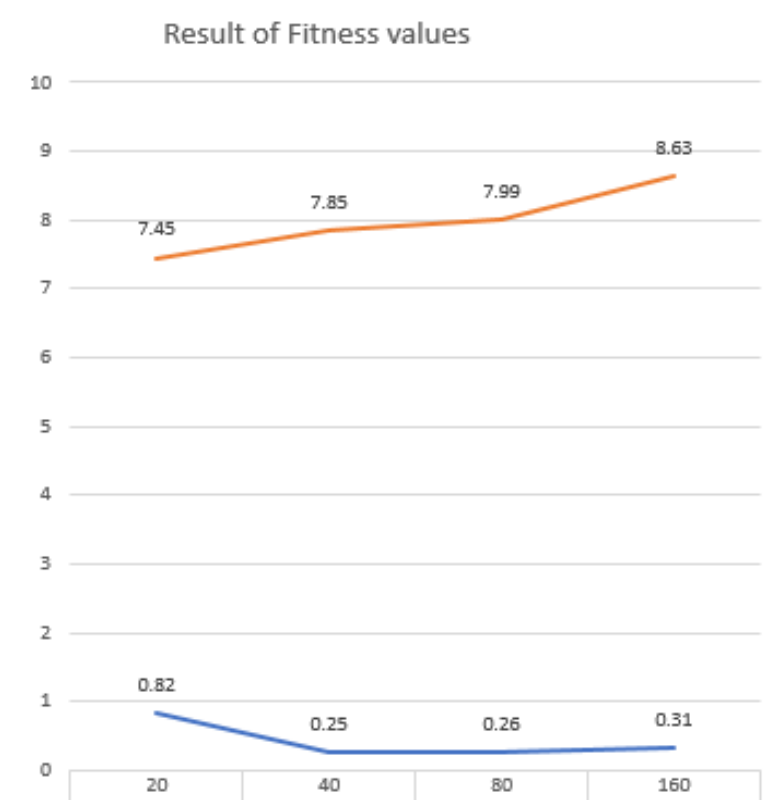

Fig 8: Fitness values with PSO and PSO+K-Means

Figure 9 below describes the purity values in a data cluster. The results obtained by using PSO and PSO + K-Means result in purity values that are not fixed. This is due to differences in test parameters being tested. Dataset in this case is very suitable when using the parameter 20 particles that produces purity 34.06 and with the smallest fitness value value is 7.45 as in figure 7 above.

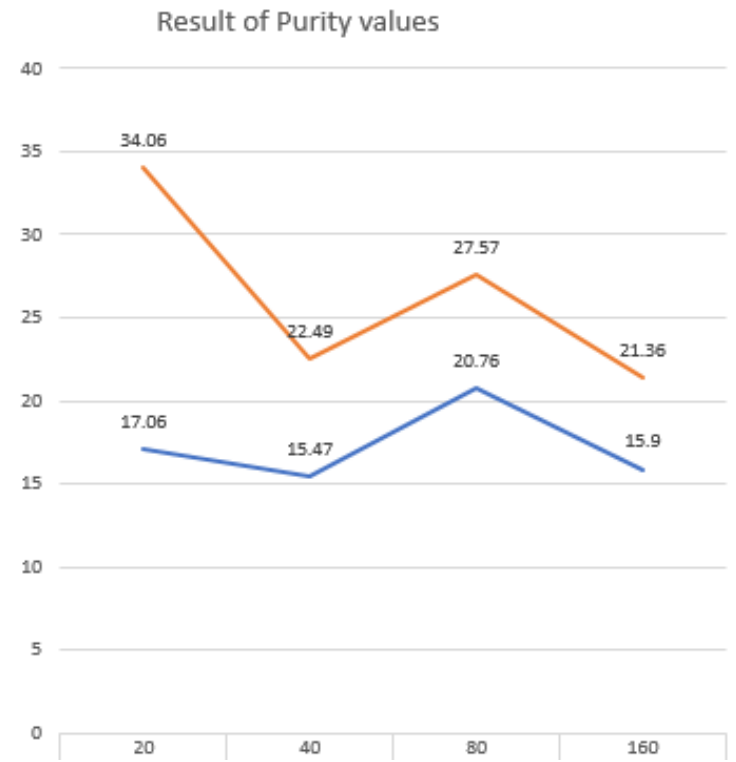

Fig 9: Purity Values with PSO and PSO+K-Means

Figure 10 below is the time comparison required to execute the dataset. The addition of particles parameters in each experiment using two different methods certainly affects the addition of time. This is due to complicated computation. 


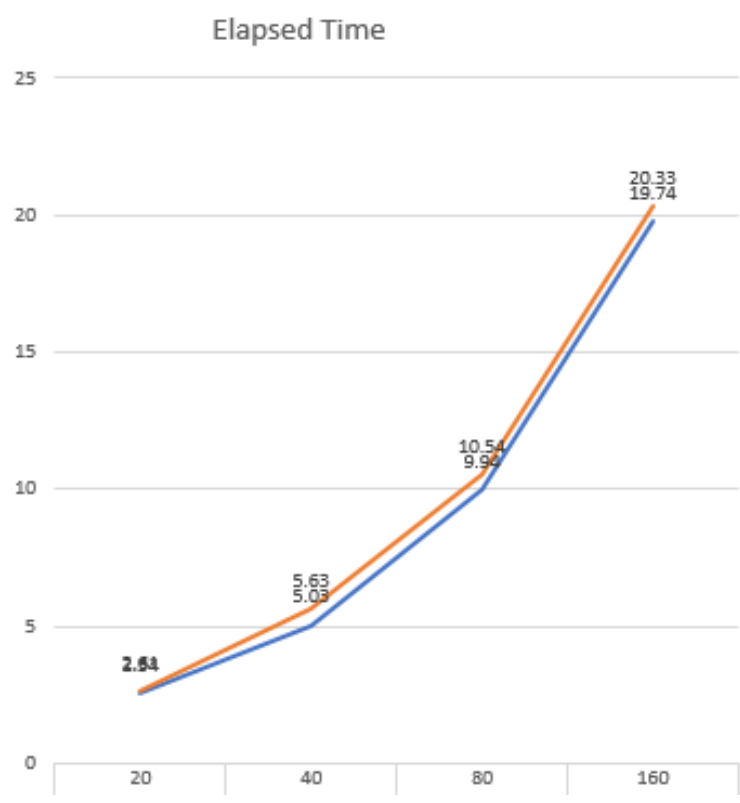

Fig 10: Elapsed Time

experimental results can be shown in the comparison table 5 below :

Tabel 5. Comparison experiment table PSO and PSO + k-Means

\begin{tabular}{|l|l|l|l|l|}
\hline Method & Particle & Fitness & Purity & $\begin{array}{l}\text { Elapsed } \\
\text { Time }\end{array}$ \\
\hline \multirow{4}{*}{ PSO } & 20 & 0.82 & 17.06 & 2.54 \\
\cline { 2 - 5 } & 40 & 0.25 & 15.47 & 5.05 \\
\cline { 2 - 5 } & 80 & 0.26 & 20.76 & 9.94 \\
\cline { 2 - 5 } & 160 & 0.32 & 15.9 & 19.74 \\
\hline \multirow{4}{*}{ PSO+k-Means } & 20 & 7.45 & 34.06 & 2.61 \\
\cline { 2 - 5 } & 40 & 7.85 & 22.49 & 5.65 \\
\cline { 2 - 5 } & 60 & 7.99 & 27.57 & 10.54 \\
\cline { 2 - 5 } & 80 & 8.65 & 21.36 & 20.33 \\
\hline
\end{tabular}

\section{CONCLUCION}

Technically the use of PSO + k-Means method is very good in the search for optimal value in a population of data, but the addition of time range of data execution will increase due to hybrid computing usage, otherwise the use of PSO method has a little data excess time span, weakness is still difficult in finding the optimal value, especially the value of purity and fitness value. In this case the use of the school dataset is suitable when using the input parameter of 20 swarm. The graphs in figure 8 and figure 9 above show that the lowest fitness value and highest purity value are in the 20 swarm parameters. The application of hybrid PSO + k-Means can provide accurate data in providing the right grouping within the implementation of school quality mapping. This method is very useful when implemented to a large dataset. Large datasets will make the use of this method more accurate, and can make calculations more effective than should be done manually. Manual calculations potentially have many errors. Therefore, the use of this method is very appropriate when applied in the education sector, school stakeholders can see how far their educational achievement.

\section{REFERENCES}

[1] H. Jiawei, K. Micheline, and P. Jian, DATA MINING (Concept and Techniques), vol. 3, no. 13. 2012.

[2] C. Cobos, H. Muñoz-Collazos, R. Urbano-Muñoz, M. Mendoza, E. León, and E. Herrera-Viedma, "Clustering of web search results based on the cuckoo search algorithm and Balanced Bayesian Information Criterion," Inf. Sci. (Ny)., vol. 281, pp. 248-264, 2014.

[3] L. Rokach and O. Maimon, The Data Mining and Knowledge Discovery Handbook. 2010.

[4] B. Mirkin, Clustering for Data Mining - A Data Recovery Approach. 2005.

[5] O. R. Vincent, A. S. Makinde, O. S. Salako, and O. D. Oluwafemi, "A self-adaptive k-Means classifier for business incentive in a fashion design environment," Appl. Comput. Informatics, 2017.

[6] M. Capó, A. Pérez, and J. A. Lozano, "An efficient approximation to the K-Means clustering for massive data," Knowledge-Based Syst., vol. 117, pp. 56-69, 2017.

[7] B. Everitt, "Cluster analysis," Qual. Quant., vol. 14, no. 1, pp. 75-100, 1980.

[8] N. Kamel, I. Ouchen, and K. Baali, "A sampling-PSO-KMeans algorithm for document clustering," in Advances in Intelligent Systems and Computing, 2014, vol. 238, pp. 45-54.

[9] B. F. . Solaiman and A. . Sheta, "Energy optimization in wireless sensor networks using a hybrid K-Means PSO clustering algorithm," Turkish J. Electr. Eng. Comput. Sci., vol. 24, no. 4, pp. 2679-2695, 2016.

[10] S. Kalyani and K. S. Swarup, "Particle swarm optimization based K-Means clustering approach for security assessment in power systems," Expert Syst. Appl., vol. 38, no. 9, pp. 10839-10846, 2011.

[11] X. Cui and T. E. Potok, "Document Clustering Analysis Based on Hybrid PSO+K-Means Algorithm," Engineering, pp. 185-191, 2005.[12] D. W. Van Der Merwe and A. P. Engelbrecht, "Data clustering using particle swarm optimization," 2003 Congr. Evol. Comput. 2003 CEC 03, vol. 1, pp. 215-220, 2003.

[12] Ahmadyfard and H. Modares, "Combining PSO and kMeans to enhance data clustering," in 2008 International Symposium on Telecommunications, IST 2008, 2008, pp. 688-691.

[13] P. Arora, Deepali, and S. Varshney, "Analysis of KMeans and K-Medoids Algorithm for Big Data," in Physics Procedia, 2016, vol. 78, pp. 507-512.

[14] B. S. Serapião, G. S. Corrêa, F. B. Gonçalves, and V. O. Carvalho, "Combining K-Means and K-Harmonic with Fish School Search Algorithm for data clustering task on graphics processing units," Appl. Soft Comput. J., vol. 41, pp. 290-304, 2016.

[15] S. J. Redmond and C. Heneghan, "A method for initialising the K-Means clustering algorithm using kdtrees," Pattern Recognit. Lett., vol. 28, no. 8, pp. 965 973, 2007. 
[16] J. Kennedy and R. Eberhart, "Particle swarm optimization," Neural Networks, 1995. Proceedings., IEEE Int. Conf., vol. 4, pp. 1942-1948 vol.4, 1995.
[17] R. C. Eberhart and Yuhui Shi, "Particle swarm optimization: developments, applications and resources," Proc. 2001 Congr. Evol. Comput. (IEEE Cat. No.01TH8546), vol. 1, pp. 81-86, 2001. 